\title{
Microstructure of Iron (Fe) Nanowires Synthesized by Chemical Reduction of Different Fe Ionic Precursors
}

Sz-Chian Liou ${ }^{1 *}$, Wen-An Chiou ${ }^{1}$, Yubing Zhou ${ }^{2}$, Xu-Xiang Cai ${ }^{3}$, Mao-Yuan Chen ${ }^{4}$, Yuh-Jing Chiou ${ }^{4}$, Hong-Ming Lin $^{3}$ and Chung-Kwei Lin $^{5}$

1. AIM Lab, Nano Center, University of Maryland, College Park, MD, USA.

2. Dep. Materials Science and Eng., University of Maryland, College Park, MD, USA.

3. Department of Materials Engineering, Tatung University, Taipei, Taiwan.

4. Department of Chemical Engineering, Tatung University, Taipei, Taiwan.

5. School of Dental Technology, College of Oral Medicine, Taipei Medical Univ., Taipei, Taiwan.

* Corresponding author: <scliou@umd.edu>

Iron (Fe)-based nanostructures are attractive applications in the areas of drug delivery, data/energy storage, catalysis due to their inexpensive price, biocompatibility and low-toxicity. The one-dimension (1D) Fe nanowires (NWs) assembled by isotropic Fe nanoparticles (NPs) with high values of anisotropy and magnetization have applications in magnetic transitions and electronic transmission due to its larger surface-to-volume ratio [1]. However, in addition to the challenge of fabricating Fe chain-like NWs without templates, few microstructure study of Fe nanowire with different valence state can be found. This research investigate the microstructure difference between Fe(II) and Fe(III) using HRTEM, electron diffraction and electron energy loss spectroscopy (EELS).

Fe nanowires were fabricated by reduction method of $\mathrm{FeCl}_{2}\left(4 \mathrm{H}_{2} \mathrm{O}\right)$ and $\mathrm{FeCl}_{3}\left(6 \mathrm{H}_{2} \mathrm{O}\right)$ as the $\mathrm{Fe}$ (II) and $\mathrm{Fe}$ (III) starting materials. The Fe starting materials were dissolved into the aqueous system with mixed $\mathrm{NaBH}_{4}$ as the reducing agent in an inert Ar gas under an external high magnetic field (2,000gauss). The details of the growth conditions can be found in previous reports [1,2]. A small drop of Fe nanowires/ethanol suspension was pipetted onto a holey $\mathrm{C}$-coated $\mathrm{Cu}$ grid. Microstructure and microchemistry were investigated with JEOL 2100F (S)TEM equipped with Gatan Image Filter (GIF, Tridiem 863). Fe Nanowires were also examined in SEM (Hitachi SU-70).

SEM (Fig. 1a and c) and TEM images (Fig. 1b and d) of Fe NWs fabricated by reduction of Fe(II) clearly shows that chain-like Fe NWs were formed by assembly of spheres-shape particles and can be grouped in two categories: one is assembled by spheres with wide range in size/diameter $(30-250 \mathrm{~nm})$ (Fig. 1a and b), whilst the other is assembled by particles with a rather uniform size/diameter $(70-140 \mathrm{~nm})$ (Fig. 1c and d). Fe(II) NWs are all crystalline as shown by HRTEM image (Fig. 1e) and ED pattern (inset in Fig. 1e). Furthermore, those spheres/particles are separated from each other by $3 \sim 5 \mathrm{~nm}$ amorphous layer (Fig. 1e), which was confirmed as an iron oxide by position-dependent EELS spectra and corresponding Fe and O mapping (Fig. 3a and b). On the other hand, morphology of Fe NWs formed by reduction of Fe (III) only appeared as chain-like NWs with a uniform size/diameter of 50-150 nm (Fig. 2a and b). Crystallinity of most Fe(III) NWs were poor or amorphous (Fig. 2b-d). Similar to the Fe(II) NWs, the 3 5nm-thick iron oxide was also observed on the surface of Fe (III) NWs (Fig. 2c, 3a and c). Morphology and particle size of Fe NWs formed by Fe(II) and Fe(III) was compared in Table 1. The final morphology and crystallinity of chain-like Fe NWs were usually formed by Fe NP-NP interactions in two steps: (1) the formation of $\mathrm{Fe} N$ Ps reduction interaction between $\mathrm{Fe}(\mathrm{II}) / \mathrm{Fe}(\mathrm{III})$ and $\mathrm{NaBH}_{4}$, and (2) the assembly of 1D chains of Fe NPs. This study confirmed that the ion mobility of $\mathrm{Fe}(\mathrm{II}) / \mathrm{Fe}(\mathrm{III})$ in solution and interface diffusion rate are two key factors in controlling the final morphology and crystallinity $[3,4]$. 
References:

[1] M Krajewski et al., Physical Status Solidi A 212 (2015), p. 862.

[2] XT Zhan et al., RSC Adv. 3 (2013), p. 15966.

[3] N Jaffrezic-Renault and SV Dzyadevych, Sensor 8 (2008), p. 2569.

[4] This work was supported by the Ministry of Sci. and Tech. of Taiwan (MOST 107-2221-E-036-004 to HML). TEM work at AIM Lab was partially supported by NSF-MRSEC (DMR 05-20471) and UMD.

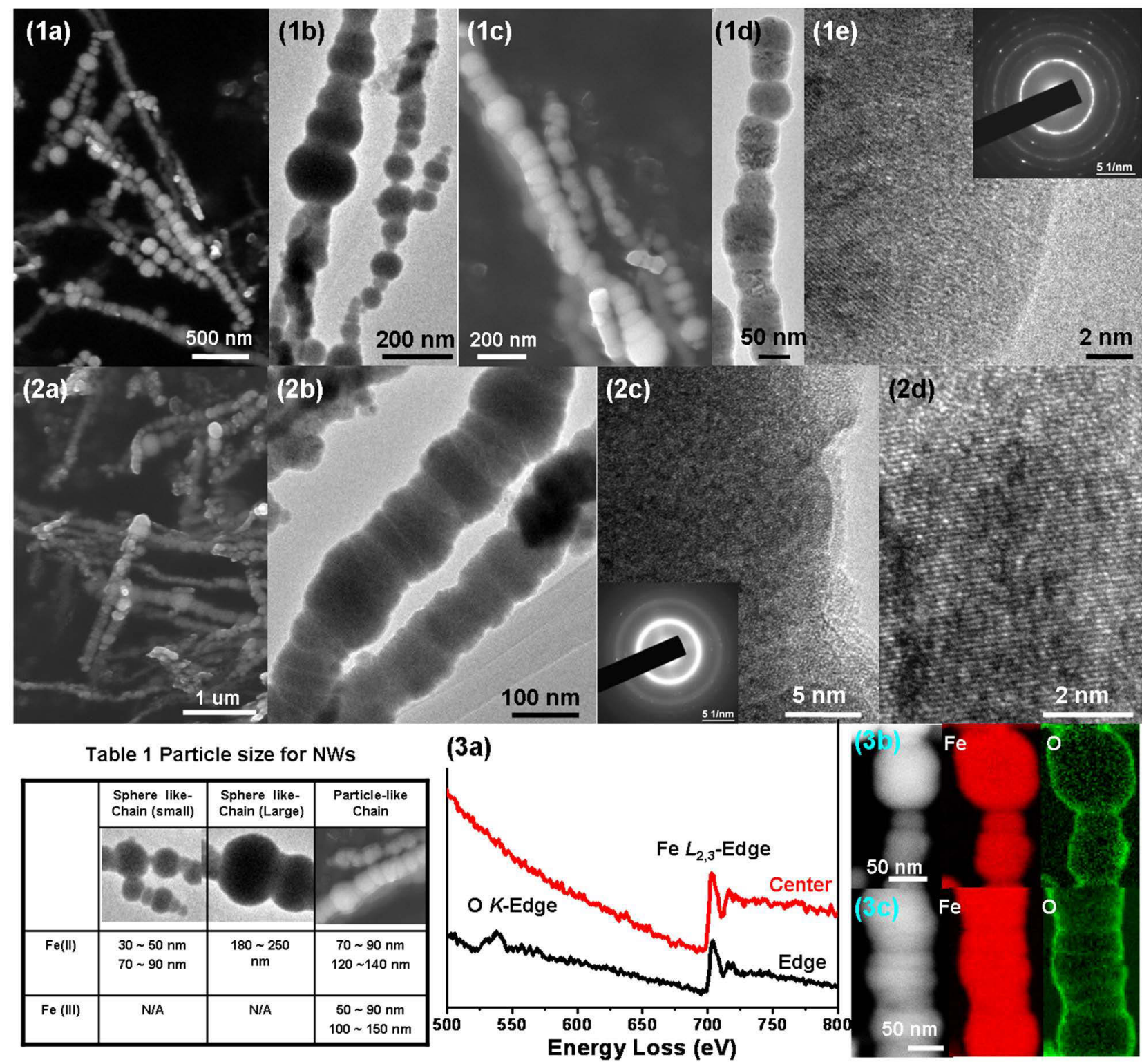

Table 1. Particle size for NWs prepared by reduction from $\mathrm{Fe}^{2+} / \mathrm{Fe}^{3+}$ precursors.

Figure 1. SEM images (a and c) and (HR)TEM images (b, $d$ and e) of Fe(II) NWs prepared by reduction from $\mathrm{Fe}(\mathrm{II})$ precursor. Inset in (e) is the ED pattern recorded from (d).

Figure 2. SEM image (a) and (HR)TEM images (b, c and d) of Fe-NWs prepared by reduction from Fe(III) precursor. Inset in (c) is the ED pattern recorded from (b).

Figure 3. (a) Position-dependent EELS spectra recorded from edge (black spectrum curve) and central position (red spectrum curve) of NPs. The HAADF images (left panel in b and c) and EELS elemental mapping of $\mathrm{Fe}$ and $\mathrm{O}$ for NPs prepared by reduction from $\mathrm{Fe}(\mathrm{II})$ (b) and $\mathrm{Fe}(\mathrm{III})$ (c). 